\section{CRYSTAL ENGINEERING OF ORGANIC-INORGANIC HYBRID SOLIDS}

A.G. Orpen

University of Bristol School of Chemistry Cantock's Close BRISTOL BS8 1TS UK.

The ability of organic moieties to participate in hydrogen bonding and other intermolecular interactions is fundamental to life and to the burgeoning field of crystal engineering. Similar functionalities are frequently present in metal complexes albeit in different electronic and steric environments. In addition metal complexes offer a range of potentially useful properties based on their electronic, optical and magnetic characteristics. We therefore have sought to establish the utility of metals and ligands in complexes for creating well defined intermolecular interactions with a view to their applications in controlling molecular aggregation. The implications for design of supramolecular interactions exploiting these characteristics will be discussed and examples of their applications to the synthesis of novel and planned crystal structures of metal complexes given.
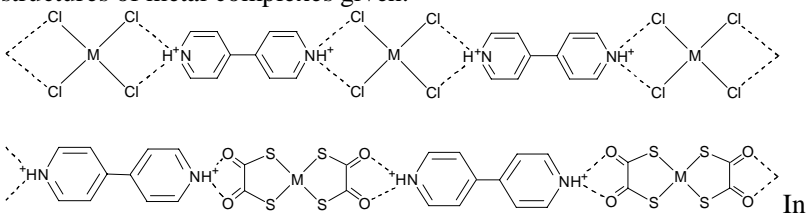

Particular a range of new crystal structures has been prepared using homoleptic anionic metal complexes with halide or dithiooxalate ligands (see above) and organic cations with NH hydrogen bond donors (see below). The effects on the structures formed of variations in the metal, and the geometries of the anion and cation will be shown.
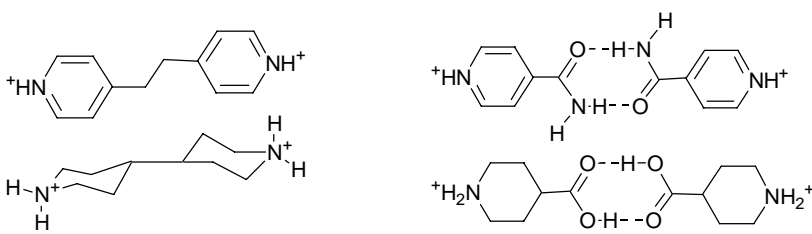

Keywords: SYNTHESIS CRYSTAL ENGINEERING SOLID STATE CHEMISTRY

\section{Acta Cryst. (2002). A58 (Supplement), C248 \\ MACROCYCLES, CATENANES AND KNOTS: SELF-ORGANIZATION OF SIMPLE BUILDING BLOCKS}

M. Nieger ${ }^{1}$ F. Voegtle ${ }^{2}$

${ }^{1}$ Department of Inorganic Chemistry, University of Bonn, Gerhard-Domagk-Str. 1, D-53121 Bonn GERMANY ${ }^{2}$ Kekule Institute of Organic Chemistry and Biochemistry, University of Bonn, Gerhard-Domagk-Str. 1, D-53121 Bonn

Supramolecular Chemistry, Self-Organization, Non-Covalent InteractionAmide type $(\mathrm{CONH})$ based macrocycles, catenanes and knots are synthesized by reaction of small building blocks, dicarboxylic acid dichlorides (e.g. isophthaloyl dichloride, pyridine-2,6-dicarbonyl dichloride, furan-2,5-dicarbonyl dichloride, etc.) and a diamine (1,1-bis(4'-amino-3',5'-dimethylphenyl)cyclohexane). In such compounds the spacer subunits (aromatic and 1,1-diphenylcyclohexyl) are connected by an amide moiety. The subunits are capable of forming hydrogen bonds and/or noncovalent interactions, which play a key role in the formation and conformation of such compounds.

Crystal structure analysis is an important tool to distinguish between macrocycles, catenanes and knots, which are constitutional isomers with identical molecular weight and formula. Depending on the solvent, the octalactam-based macrocycles show different conformations and hydrogen-bonding patterns. Crystallographic determination was found essential in characterizing the isomerism of catenanes (e.g., in/out, out/out or in/in) and comparative evaluation of the hydrogen-bonding patterns in these compounds. [1] The structure of the first molecular knot with twelve amide groups was determined for the first time by structure analysis. These knots behave like small proteins with around $30 \%$ partly disordered solvent in the unit cell. Structure determination by common structure solution programs using default parameters failed. The intramolecular hydrogen-bonding pattern is similar to that one recently found in amide-based [2] rotaxanes: a novel type of selforganization by internal templating reaction. [2] The chiroptical properties and chirality can be explained on the molecular level of the crystal structure and on the basis of theoretical calculations.

References

[1] Q. Y. Li, E. Vogel, A. H. Parham, M. Nieger, M. Bolte, R. Fröhlich, P.Saarenketo, K. Rissanen, F. Vögtle, Eur. J. Org. Chem. 2001, 4041-4049.

[2] O. Safarowski, M. Nieger, R. Fröhlich, F. Vögtle, Angew. Chem. Int. Ed. Engl. 2000, 39, 1616-1618.

Keywords: SUPRAMOLECULAR CHEMISTRY SELF ORGANISATION NON-COVALENT INTERACTION
Acta Cryst. (2002). A58 (Supplement), C248

$\underline{\text { K.T. Rissanen }}$

Department of Chemistry, University of Jyväskylä, Finland

Supramolecular Chemistry[1] studies weak non-covalent interactions such as $\mathrm{H}$-bonding, $\pi \ldots \pi-, \mathrm{CH} / \pi$-interactions, hydrophobic effects etc., between two or more molecules and during the last ten years a concept of using noncovalent supramolecular interactions to create (synthesize) large molecular structures has emerged. of particular interest have been the associations induced by $\mathrm{H}$-bonding or metal coordination, to create large spherical or helical molecular assemblies. Highly sophisticated ligands ("programmed" molecular components)[1] or suitable suitably functionalized compounds can be connected together by weak non-covalent interactions, creating reversible molecular entities. Functionalized calixarenes and resorcinarenes[2], have been recently found to be excellent, quite easily available, structural components for spherical dimers, viz. capsules, or even larger assemblies. Resorcinarenes can be further functionalized and other types of H-bonded assemblies can be created. Hydrogen bonding can also be used to construct dendritic assemblies and when crystalline, allowing structural determination of the weak intermolecular interactions. The lecture will focus on resorcinarenes and their derivatives as building blocks for $\mathrm{H}$-bonded capsules and also introduces $\mathrm{H}$ bonded analogues of cavitands, together with some examples of other hydrogen bonded molecular assemblies.

References

1. J.-M. Lehn, Supramolecular Chemistry, VCH, Weinheim, 1995.

2. P. Timmerman, W. Verboom, D. N. Reinhoudt, Tetrahedron 1996, 52, 26632704.

Keywords: SUPRAMOLECULAT ASSEMBLIES, HYDROGEN BONDING, WEAK INTERACTIONS

\section{Acta Cryst. (2002). A58 (Supplement), C248}

\section{SUPRAMOLECULAR WIRE-TYPE CONFINEMENTFOR IODINE MOLECULES IN AN ORGANIC ZEOLITE}

T. Hertzsch $^{1,2}$ F. Budde ${ }^{1}$ E. Weber ${ }^{2}$ J. Hulliger $^{1}$

${ }^{1}$ University of Berne, Department of Chemistry and Biochemistry, Freiestrasse 3, CH-3012 Berne, Switzerland ${ }^{2}$ TU Bergakademie Freiberg; Institute of Organic Chemistry, Leipziger Strasse 29, D-09599 Freiberg, Germany

One-dimensional arrays of atoms or molecules showing the property of conducting charge or photonic energy are considered major components for devices based on molecular sized functional units. Here, we report on the synthesis of co-crystals of tris(o-phenylenedioxy)cyclotriphospazene (TPP) and iodine as well on conductivity properties of single crystals of TPP- $\left(\mathrm{I}_{2}\right) \quad(\mathrm{x}=$ 0.65-0.75) measured parallel and perpendicular the channel axis of the organic zeolite (Angew. Chem. (2002), in press). Loading by iodine can by achieved by (i) exchanging a template used to form the zeolite structure, or (ii) by cocrystallization of TPP and iodine in mesitylene. (i) Millimeter long crystals of TPP-(THF) $)_{0.6}$ (THF: tetrahydrofuran) were obtained by lowering the temperature of a saturated solution in THF at $60{ }^{\circ} \mathrm{C}$. Exchange of THF by iodine was observed already at $20^{\circ} \mathrm{C}$, however, effective exchange was performed by heating TPP(THF) ${ }_{0.35-0.6}$ crystals in ampoules containing iodine. (ii) Co-crystallization of TPP and iodine in mesitylene has produced TPP-(I)x crystals showing the maximum of theoretical loading of 0.75 . In TPP-( $\left.\mathrm{I}_{2}\right)_{0.7}$ crystals iodine form chains in the channels build up by phenyl rings of TPP. The dark conductivity $\sigma$ (parallel) varied in the range of $10^{-6}$ to $10^{-8} \Omega^{-1} \mathrm{~m}^{-1}$. The upper limit of present values is of the same order as the conductivity in the (b,c)-plane of crystalline iodine. An anisotropy factor of $\sigma$ (parallel)/ $/$ s(perpendicular) of about 30 provides evidence for a preferred conductivity along iodine chains in TPP channels. Summarizing, we present a first example where iodine molecules were brought into a chain-like configuration.

Keywords: ORGANIC ZEOLITE, INCLUSION COMPOUNDS, MOLECULAR WIRE 